Bolm. Zool., Univ. S. Paulo $9: 177-193,1985$

\title{
MIGRACÃO VERTICAL DE LUCIFER FAXONI BORRADAILE, 1915 (CRUSTACEA-DECAPODA) NAS ÁGUAS AO LARGO DE SANTOS, BRASIL
}

MARIA PALOMA JIMENEZ ALVAREZ

Departamento de Zoologia, Instituto de Biociênsias, Universidade de São Paulo, Caixa Postal 20520, 01498 - São Paulo Brasil

(recebido em 14.II.1985).

RESUMO - A migração vertical dos vários estágios de desenvol vimento de Lucifer faxoni foi estudada nas quatro estaçōes do ano, em äguas ao largo de Santos (24016's e 46000' W). Os espécimes maduros de ambos os sexos migraram segundo o mesmo padrão. Houve diferenças na migração de espécimes maduros e larvas em julho (1960) quando as larvas migram inversamente, e em novembro (1961) quando elas se concentraram próxi mas à superfície. A distríbuição vertical da espécie em a bril (1960) foi limitada possivelmente pela presença de ägua fria abaixo dos 20 metros de profundidade.

ABSTRACT - The vertical migration of several developmental stages of Lucifer faxoni was studied off Santos (24016'S and $46000 \mathrm{~W}$ ) in four different seasons. The mature specimens of both sexes migrated similarly. There were differences in the migration of mature and larval stages in July, 6 (1960) when the larvae migrated inversely, and in November, 7 (1961) when they concentrated near the surface. The vertical distribut ion of the species in April, 8 (1960) was possibly limited by the presence of cold water below $20 \mathrm{~m}$ depth.

\section{INTRODUÇÃO}

A migração vertical do plâncton ao largo de Santos vem sendo estudada por diversos autores em diferentes grupos de animais: Copépodos (Bjorrnberg, 1969), Chaetognatha (Almeida Prado, 1968), Hydromedusae (Moreira, 1973), plâncton total (Moreira, 1976), Appendicularia (Sinque, 1976), Ostracoda (Rocha, 1982) e Cladocera (Rocha, 1983).

o estudo da migração vertical de Lucifer faxoni visa contribuir para uma melhor compreensão das relaçōes ecolōgicas das migraçōes, o que só é possível com o estudo detalhado das especies presentes no pláncton (Moreira, 1976).

Luoifer faxoni é um elemento tipicamente costeiro e de águas de plataforma, frequente em estuărios e regiōes laguna res (Rodriguez, 1966 e 1973; Sandifer, 1973). Em àguas brasí 
leiras Costa \& Prandi (1971) não registraram a presença de Lucifer faxoni em águas com salinidade acima de $36 \%$. Harper (1968) registrou a espécie nas äguas da costa do Texas com salinidade de $37,3 \%$. Embora geralmente citado como planctônico e como un dos "poucos crustáceos-decāpodos holoplanctônicos" (Harper, 1968) também foi coletado dragando-se fundos lodosos (Brooks, 1882 ; Woodmansee, 1966a).

Sua distribuição geogrấfica é muito ampla, tendo sido encontrado nos Oceanos Indico, Pacífico e Atlântico. Na costa brasileira ocorre desde a foz do Rio Pará até a Lagoa dos Patos (Barth, 1963; López, 1966)

Nas águas brasileiras foi primeiramente estudado por Barth (1963 e 1964) quanto à distribuição horizontal. Costa \& Prandi (1971) estudaram a distribuição vertical e também fizeram uma revisão sistemática das duas espécies ocor rentes em nossas äguas. 0 ciclo de vida de Lucifer foi estudado em Cananéia, litoral sul do Estado de São Paulo, por Ló pez (1966).

Woodmansee (1966a) estudou a migração vertical de $L$. faxoni no Golfo do México e a relacionou, bem como o trans porte horizontal, com o ciclo solar e com as marés.

Woodmansee $(1966, \mathrm{~b})$ analisou também a migraçã̄o verti cal de fémeas na época de reprodução, verificando que estas nadam para a superfície justamente antes da eclosão, deixando os náuplios na superficie, fato este que já havia sido ob servado por Brooks (1882)

Experimentalmente foi verificado para vários crustáceos que a influência da luz na migração vertical pode ser alterada pela temperatura (Cushing, 1951). A temperatura é un fator limitante efetivo no controle da migração vertical de muitas espécies e especialmente a termoclina pode atuar como uma barreira eficaz na distribuição vertical do zoo plâncton.

Como as águas da costa brasileira recebem a influência da água fria de origem antártica que permanece no fundo sepa rada das äguas quentes superficiais por uma ternoclina, pare ceu interessante estudar a influência da temperatura na distribuição de Lucifer e verificar se individuos de sexos dife rentes e em diferentes estägios de desenvolvimento tem pa drōes semelhantes de migração.

\section{MATERIAL E MÉTODOS}

As amostras da Coleção de Plâncton, série V, utiliza das foram coletadas ao largo de Santos ( $24016^{\prime} \mathrm{S}$ e $46^{\circ} 00^{\prime} \mathrm{W}$ ) pe la Dra. Maria da Glória Soares Moreira, nos dias 8/4/1960, 22/9/1960, 6/7/1961 e 7/11/1961.

Os dados seguintes sobre as amostragens são descritos por Moreira (1970 e 1973).

As amostras de plâncton foram obtidas com uma rede de fechamento tipo Standard no $3(0,50 \mathrm{~m}$ de diâmetro de boca $1,80 \mathrm{~m}$ de comprimento e 64 malhas por polegada) A rede foi arrastada a cada 4 hs, no intervalo de 24 hs, durante $15 \mathrm{mi}-$ nutos sucessivamente em cada um dos três níveis: superfície (1 m), um pouco acima do fundo $(45-47 \mathrm{~m})$ e à meia-ăgua (20- 
$25 \mathrm{~m}$ ) A velocidade de arrasto foi mantida tão próxima quanto possivel de 0,5 nó o que significa $156 \mathrm{~m}$ percorridos em 15 minutos. A quantidade de àgua do mar filtrada foi estimada pela fôrmula $r^{2} . h$, sendo $r$ o raio da boca da rede $e h$ a distancia por ela percorrida. Todas as amostras de plâncton foram fixadas com formalina a $10 \%$

Para a determinação da temperatura, coletaram-se em ca da estação amostras de água com garrafa de Nansen.

A salinidade foi titulada no laboratório pelo método de Knudsen.

A temperatura e a salinidade foram determinadas em pro fundidades estandartizadas. Nos gráficos a temperatura e salinidade sāo representadas pela média diária de cada sërie em cada profundidade. As horas indicadas nas figuras cor respondem sempre ao infcio de uma sẻrie de coletas.

Todos os exemplares foram retirados das amostras to tais para contagem, determinação do sexo, do estăgio de de senvolvimento, maturidade e identificação das várias fases larvais.

A identiflcação da espécie e a determinação do sexo fo ram feitas segundo a descrição de. Hansen (1919), Bowman(1967) e Bowman \& McCain (1967) Para verificar o estágio de desenvolvimento foram tomadas medidas do somito pré-bucal, as quais têm uma relação direta com o comprimento total dos indivỉduos (López, 1966 e Woodmansee, 1966a). 0 comprimento do somito prè-bucal usado é a distância da margem anterior do labro até a base do pedúnculo ocular.

Todas as formas pós-larvais foram medidas e separadas em quatro categorias: fêmeas maduras, machos maduros, machos imaturos e individuos imaturos. Foram consideradas fêmeas ma duras as que apresentaram ovários visiveis à lupa Baush \& Lomb com ou sem coloração e aumento até 60 vezes. O corante usado foi o carmim borácico Grenacher segundo o processo de Peacock (1966).

Considerou-se macho maduro aquele que apresentou: es permatôforo, petasma bem desenvolvido com bainha e dois espi nhos no sexto segmento abdominal. Machos imaturos são aque les sem eśpermatóforo, com petasma pequeno com extremidade livre ou encurvada, sem balinha envolvendo-o e com os dois es pinhos do sexto segmento abdominal ausentes ou já um poucō proeminentes. Como individuos imaturos consideraram-se todos aqueles que não apresentaram as características de indiví duos maduros e de machos imaturos. Nos grấficos e tabelas a categoria de imaturos inclui sempre os machos imaturos com os demals espécimes não sexuados. Não foi possivel diferen ciar as fêmeas imaturas atravês do diámetro do orificio geni tal como foi feito por López (1966), por estarem as amostras preservadas há muito tempo.

As fases larvais foram separadas em três grupos segundo o estudo de Brooks (1882): protozoea, zoea e esquizópodo. Na análise hidrográfica seguiu-se a classificação das massas de água feita por Emílson (1961) para a região entre Cabo Frio (lat 22055') e o Estado de Santa Catarina (lat. $28^{\circ} 00^{\prime}$ ) e os resultados de Mesquita (1974) 
As quatro massas de água que poden ocorrer na região en estudo são:

1. Agua tropical:de salinidade maior que 36to e temperatura maior que $20^{\circ} \mathrm{C}$. E fornada ao largo da costa leste do Brasil devido à intensa radiaçāo e evaporaçāo.

2. Agua subtropical: de salinidade 35-36\% e temperatura entre 10 e $20^{\circ} \mathrm{C}$. Resulta da mistura entre água tropical e água de baixa salinidade e temperatura vinda do sul.

3. Igua costeirg: de salinidade menor que 35\% e temperatura maior que $20^{\circ} \mathrm{C}$. E formada próxima à costa por aquecinento local e por nistura de água doce.

4 Agua de plataforma: de salinidade 35-36\% e temperatura maior que $20^{\circ} \mathrm{C}$. E formada sobre a plataforma por nistura de água tropical, subtropical e costeira, influenciada por aquecimento local.

Tabela I - Massas de água presentes nos quatro períodos estu dados nas várias profundidades amostradas.

\begin{tabular}{lllll}
\hline $\begin{array}{l}\text { Profun } \\
\text { didadē } \\
\text { (m) }\end{array}$ & $8-9 / 4 / 1960$ & $22-23 / 9 / 1960$ & $6-7 / 7 / 1961$ & $7-8 / 11 / 1961$ \\
\hline $0-7$ & Plataforna & Plataforna & Costeira & Plataforma \\
\hline 7-15 & Tropical & $\begin{array}{l}\text { Tropical } \\
\text { de }\end{array}$ & Plataforma & Subtropical \\
\hline $25-47$ & Subtropical & Tropical & Tropical & Subtropical \\
\hline
\end{tabular}

\section{RESULTADOS}

Foram estudados 14481 exemplares de Lucifer fasoni per tencentes aos diversos estágios de desenvolvimento. Na Tabela II é dado o número de indivíduos en $100 \mathrm{~m}^{3}$ de àgua, para as quatro séries estudadas.

8 de abril de 1960 : (Figs. 1 a 5)

Do total de 1126 exemplares a maior porcentagen foi de larvas (578), principalmente na fase de protozoea e en segui da de machos maduros (278).

As maiores densidades foran registradas na superfície à noite e ao nível de 20 netros durante o dia. A migraçāo vertical restringiu-se portanto à faixa acina dos 20 metros, enbora alguns machos maduros, inaturos e larvas tenhan alcan çado o fundo.

Os vários estágios de desenvolvinento da espécie, estú dados separadamente, migraram segundo esse padräo.

Os estágios larvais ocorreran nos trés níveis porén 
preferencialmente na região da termoclina (acima dos $20 \mathrm{~m}$ de profundidade).

Nota-se uma preferéncia pela região com água de plataforma e ägua tropical porém a espécie evitou a região do fun do com temperatura de $16,5^{\circ} \mathrm{C}$ aproximadamente.

22 de setembro de 1960: (Figs. 6 a 10)

Neste período ocorreram 1903 indivíduos dos quais a me tade $(53,78)$ eram estágios larvais.

Os indivíduos maduros ocorreram preferencialmente no fundo e em pequena quantidade à meia-água, durante o dia. No ta-se una ascensão noturna gradual para a superfície porém sem uma ausência total da espécie nos níveis inferiores.

Os indivíduos imaturos e as larvas ocorreram às 16 hs em maior número na superfície do que nos níveis inferiores Só alcançaram a superfície durante a noite as larvas na fase de esquizópodos, as quais tambèm predominaram à meia-ăgua às 8 e 12 hs.

A espécie ocorreu nas massas de água presentes na re gião: água de plataforma na superfície e água tropical abaixo dos 15 metros.

6 de julho de 1961: (Figs. 11 a 15)

Esta série foi a que apresentou maior número de exem plares perfazendo 6506 indivíduos, dos quais 6261 foram espé cimes em estágio larval, indicando que nesta época tinha ocorrido reprodução acentuada da espécie. Das fases larvais a maior porcentagem foi de protozoeas.

Os indivíduos maduros e imaturos de modo geral migra ram verticalmente da superfície ao fundo.

As larvas permaneceram preferencialmente no fundo e à meia-água realizando una migraçāo inversa. A maioria das lar vas que alcançaram a superficie à noite estavam na fase dē esquizópodo.

Adultos e imaturos ocorreram nas três massas de água registradas porém a distribuiçāo das larvas foi principalmen te nas águas de plataforma e tropicais.

7 de novembro de 1961: (Figs. 16 a 20)

Nesta data foi registrado o menor número de exemplares, is to $e^{\prime}, 946$ indivíduos. As larvas foram os indivíduos mais abundantes e a fase de esquizópodo foi predominante.

$0 s$ indivíduos maduros e os imaturos dos dois sexos ocorreram em maior número na superfície à noite às 8 e às 16 hs, e às 12 hs no fundo ou à meia-água.

As larvas e imaturos foram mais abundantes na superfície, em águas de plataforma, acima da termoclina.

\section{DISCUSSR̃o}

A espécie migrou verticalmente da superfície ao fundo em 22/9/60, 7/11/6I e 7/7/61 e da superfície aos 20 metros de profundidade em $8 / 4 / 60$. 
Lucifer faxoni foi encontrado tanto acima como abaixo da termoclina, portanto isoladamente a termoclina não foi um fator limitante para a distribuição da espécie.

Os indivíduos dos dois sexos migraram segundo padrões semelhantes.

As värias fases larvais acompanharam o padrão de migra ção dos adultos em duas estaçōes (abril de 1960 e setembro de 1960), mas em julho de 1961 migraram inversamente e em no vembro de 1961 concentraram-se perto da superfície.

A ascensão das fêmeas maduras para o nível de 25 me tros, e a presença das larvas na superfície às 12 horas em 7 de novembro de 1961 talvez possa ser relacionada com 0 fato do tempo estar encoberto. Moreira (1970 e 1973) verificou também essa exceção para o plâncton total e para cinco espécies de medusas (Ectopleura dumortieri, Euphysora gracilis., Turritopsic nutricula, Proboscydactyla ornata e liriope te traphyla). 0 osträcodo Euconohoecia chierchiae também foi registrado na superfície nessa ocasião (Rocha, 1982) Nesta mesma data os indivíduos maduros às 4 horas jă haviam inicia do o movimento descendente, concentrando-se aos 25 metros, porém às 8 horas estavam novamente na superfície. Moreirá (1973) obteve o mesmo quadro para as medusas citadas aci ma. Dos fatores registrados no momento da coleta nesse dia o único que variou foi o vento, o qual vinha do sul às 4 horas e passou a soprar do leste às 8 horas, podendo assim ter trazido uma população diferente de Lucifer para o local onde se faziam as coletas.

A concentração dos imaturos e principalmente das fases larvais na superfície que ocorreu em novembro de 1961, das 12 às 8 horas finais, pode estar relacionada com a grande abundância de fitoplâncton na profundidade de 25 metros que teria atuado como uma barreira. Há evidências de que o fitoplâncton deve ter algum efeito na migração vertical de crustáceos planctontes. Um excesso de fitopláncton teria um efei to depressivo sobre o zooplâncton por secretar substâncias tóxicas segundo Hardy \& Gunther (1935) Hä algumas indica ções que possivelmente fundamentam essa idéia (Lucas, 1936 ; Bainbridge, 1953; Ryther, 1954) 0 efeito tóxico nāo é exten sivo a todas as espécies de algas planctônicas (Bainbridge, 1953) Trabalhos experimentais sobre essa influência na mi gração horizontal de filtradores em culturas de flagelados e diatomáceas, indicam que o fator relacionado pode ser algu mas vezes a substância secretada na água e outras vezes a presença física real das células vegetais (Bainbridge, 1949, 1953 e 1961). Possivelmente o efeito mais direto das diatoma ceas pode resultar do muco por elas secretado. Tem sido suge rido que tal muco pode alterar a viscosidade da água o sufíciente para diminuir consideravelmente a taxa de afundamento de ovos e possivelmente também afeta a locomoção dos outros estägios (Maloney \& Tressler, 1942 em Bainbridge, 1961)

Nas duas séries sem termoclina $(22 / 9 / 60$ e $6 / 7 / 61)$ a es pécie migrou da superfície ao fundo.

Em setembro machos e fêmeas foram mais frequentes aos 47 metros de profundidade e à meia-ägua. A haloclina presente entre 15 e 25 metros pode ter levado os indivíduos madu - 
ros a preferir as camadas profundas, de condições mais estāveis, apesar de terem temperatura inferior.

Em julho as larvas migraram inversamente aos padrões seguidos pelas outras fases de Lucifer. Bainbridge (1961) ci ta várias espécies que migram de maneira inversa do que aqué la comumente encontrada. Tais migrações inversas são conhecí das para Acartia clausi, A. Zongiremis, Nyctiphanes couchii, Evadne sp., Oithona nana, Daphnia lumholsi, estägios IV, V e adultos de Calanus finmarchicus, Diaptomus banforanus e Cyclops bicuspidatus. Moore (1950) obteve indicações de que o eufausiáceo stylocheiron migra inversamente no Mar Mediterrâneo. Moore \& O'Berry (1957) encontraram três espécies de copépodos que apresentaram migração inversa: Calocalanus pavo, Corycaeus furcifer e $C$. elongatus. Evadne nordmanii migra inversamente no Mar da Irlanda nos estudos de Lee (1974)

Foram encontradas diferenças na hora de início do movi mento ascendente para individuos maduros em relação a imatu ros e larvas. Em abril e setembro os imaturos e larvas ini ciaram o movimento para a superfície antes dos maduros (16 e 20 horas respectivamente)

0 movimento descendente de imaturos e larvas também foi anterior ao dos indivíduos maduros nos meses de abril e setembro. Cushing (1951) refere-se ao tubo experimental de Steuer (1902) no qual náuplios e jovens de Cyclops frequente mente chegaram à superfície mais rapidamente do que os aduI tos. Isto também foi verificado para Diaptomus shoshone (Pennak, 1944, em Cushing, 1951) e para jovens de Sagitta elegans (Farran, 1947, em Raymont, 1963)

AGRADECIMENTOS - Agradeço à Dra. Tagea K.S. Björnberg a revi são e as sugestões apresentadas a este estudo. À Dra. Glória Soares Moreira pela coleta do material.

\section{REFERENCIAS}

ALMEIDA PRADO, M.S. de A. 1968. Distribution and annual occurrence of Chaetognatha off Cananéia and Santos Coast (São Paulo, Brazil). Bolm Inst.Oceanogr., S. Paulo, 17 : 33-55.

BAINBRIDGE, R. 1949. Movement of zooplankton in diatom gra dients. Nature, $163: 910-911$.

BAINBRIDGE, R. 1953. Studies on the interrelationships of zooplankton and phytoplankton.J.Mar.biol.Ass.U.K., 32 (2):385-447

BAINBRIDGE, R. 1961. Migrations. In WATERMAN, Talbot H., Ed. The physiology of Crustacea. New York, Academic Press,v. $2: 431-463$.

BARTH, R. 1963. Estudos sobre Lucifer reynaudi como indica dor de águas tropicais. Notas Tecn.Inst.Pesq.Mar.Rio de J., $12: 1-16$, lâm. 1-15.

BARTH, R. 1964. Observações em indicadores biológicos na cor rente do Brasil. Anais Acad.bras.Ciênc., 36(2):217-225. 
BJORNBERG, T.K.S. 1969. Distribution of planktonic Copepods in Shelf Waters off Santos. IN: Simpósio Intemacional so bre a Fertilidade do Mar, São Paulo, 1 a 6 de Dezembro dé 1969. Resumos. São Paulo, 53 p., p. 19.

BOWMAN, T.E. 1967. The planktonic shrimp, Lucifer chacei sp. nov., the Pacific twin of the Atlantic Lucifer faxoni. Pac.Sci. 21(2):266-271.

BOWMAN, T.E. \& J.C. MCCAIN 1967. Distribution of planktonic shrimp, Lucifer in the western North Atlantic. Bull. of Mar. Science, $17(3): 660-671$.

BROOKS, G. 1882 . Lucifer, a study in morphology. Phil. Trans. R. Soc., $173(1): 57-137$, pls $1-11$.

COSTA, H.R. \& N.R.W. PRANDI 1971. Estudo da distribuição das espécies do gênero Lucifer em amostras de plâncton coleta das entre Cabo Frio e a Baía de Santos. Archos Mus.nac. , Rio de $J$., 54:159-170.

CUSHING, D.H. 1951. The vertical migration of planktonic Crustacea. Biol.Rev., $26(2): 158-192$.

EMILSON, I. 1961. The shelf and coastal waters off Southern Brazil. Bolm Inst.Oceanogr., S.Paulo, 11(2):101-112.

HANSEN, H.J - 1919. The Sergestidae of the Siboga Expedition. Siboga Exped., Mon. 38:48-65, 1ām. 4, figs 6, 7,8, lám. 5 , figs $2,3,4$.

HARDY, A.C. E.R. GUNTHER 1935. The Plankton of the St. Georgia whaling ground and adjacent waters 1926-7 Discovery Rep., $11: 1-377$

HARPER, D.E. Jr-1968. Distribution of Lucifer faxoni (Crustacea; Decapoda; Sergestidae) in neritic waters off the Texas coast, with a note on the ocurrence of Lucifer ty pus. Contr.mar.Sei., 13:1-16.

HARRIS, J.E. 1953. Physical factors involved in the vertical migration of zooplankton. Q.J2.microse.Sei., 94:537-550.

LEE, J.W. 1974. The vertical distribution and diurnal migration of Cladocera, Evadne nordmanii Lovén at different stations in the Irish Sea. J.Oceanol.Soc., Korea, 9 (1-2) $: 1-9$.

LOPEZ, M.T. 1966. Biologia de Lucifer faxoni, Borradaile 1915, en Cananéia, Brasil (Crustacea, Decapoda, Luciferidae). Bolm Inst.Oceanogr., S.Paulo, 15(1):47-54.

LUCAS, C.E. 1936. On certain interrelations between phyto plankton and zooplankton under experimental conditions J.Cons perm. int.Explor.Mer., 2:343-362.

MALONEY, M.T W.L. TRESSLER 1942. The diumal migration of certain species of zooplankton in Caroga Lake, New York Trans.Am.microscop.Soc. 61:40-51.

MESQUITA, A.R. 1974. Report on the seasonal variations of coastal waters, Brazil (Lat. $24^{\circ}$ ). I. Relatório interno, Inst. Oceanogr. Univ. S.Paulo, 1:1-36.

MOORE, H.B. 1950. The relation between the Euphausiacea and the scattering layer. Biol.Bul2,, $99(2): 181-212$.

MOORE, H.B. O'BERRY, D.L. 1957. Plankton of the Florida current. IV Factors influencing the vertical distribution of some common copepods. Bull.mar.Sci. Gulf Caribb., 7(4) :289-315.

MOREIRA, M.G.B.S. 1970. Fisio-ecologia de hidromedusas (Cni- 
daria-Hydrozoal do Atlântico Sul Ocidental. Tese de Douto ramento do Depto de Fisiologia Geral do Instituto de Bio= ciências da Univ. de S. Paulo, 99 p.

MOREIRA, M.G.B.S. 1973. On the diurnal vertical migration of Hydromedusae off Santos, Brazil. Publs.Seto mar.Lab., 20 : 537-566.

MOREIRA,M.G.B.S. 1976. Sobre a migração vertical diária do plâncton ao largo de Santos, Estado de São Paulo. Brasil. Bolm Inst.oceanogr., S.Paulo, $25(1): 55-76$.

PEACOK, H.A. 1966. Elementary Microtechnique. London, Edward Arnold LTDA, $3^{\text {rd }}$ edition, 547 p.

RAYMOND, J.E.G. 1963. Plankton and productivity in the oceans. New York, Pergamon Press, vii, 660 p.

ROCHA, C.E.F 1982. Distribution of the marine Cladocerans (Crustacea, Branchiopoda) off Santos, Brazil. Bolm Zool., Univ. S.Pauzo, 7:155-169.

ROCHA, C.E.F 1983. Distribution of Euconchoecia chierciae G.W. Muller (Crustacea, Ostracoda) off Santos, Brazil

Bolm Zool., Univ. S.Paulo, 6:53-61.

RODRIGUEZ, G. 1966 Estudios Hidrobiologicos en el Estuario de Maracaibo. Instituto Venezolano de Investigaciones $\mathrm{Ci}-$ entificas, $150 \mathrm{p}$.

RODRIGUEZ, G. 1973. El sistema de Maracaibo. Instituto Venezolano de Investigaciones Cientificas, $395 \mathrm{p}$.

RYTHER, J.H. 1954. Inhibitory effects of phythoplankton upon feeding of Daphnia magna with reference to growth, reproduction, and survival. Ecology, 35:522-533.

SANDIFER, P.A. 1973. Distribution and abundance of Decapod Crustacean larvae in the York River Estuary and Adjacent Lower Chesapeake Bay, Virginia, 1968-1969. Chesapeake Sci., $14(4): 235-257$

SINQUE, C. 1976. A migracāo vertical das Appendicularia (Tunicata) ao largo de Santos (Brasil) São Paulo, USP, $69 \mathrm{p}$. (Tese de Mestrado apresentada ao Departamento de Zoologia do Instituto de Biociências, USP).

WOODMANSEE, R.A. 1966a. Daily vertical migration of Lucifer. Planktonic numbers in relation to solar and tidal cycles. Ecology, 47(5):847-850.

WOODMANSEE, R.A. $1966 \mathrm{~b}$. Daily vertical migration of Lucifer. Egg development, oviposition and hatching. Int.Revue ges. Hydrobiol., 51(5):689-698. 
年

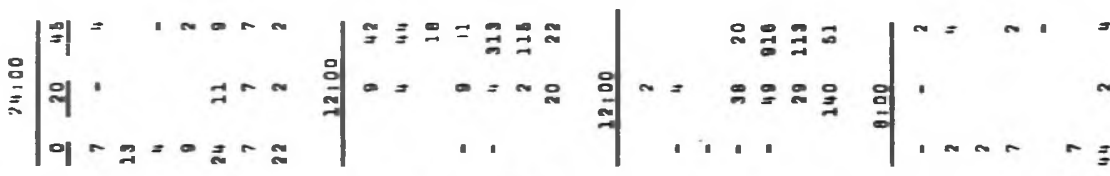

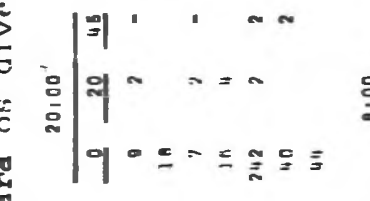

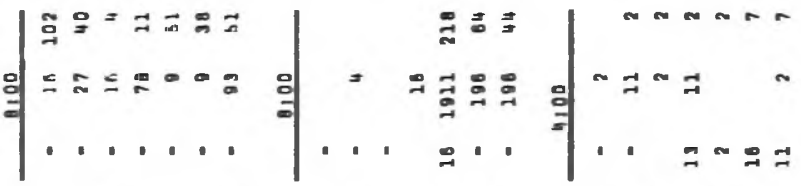

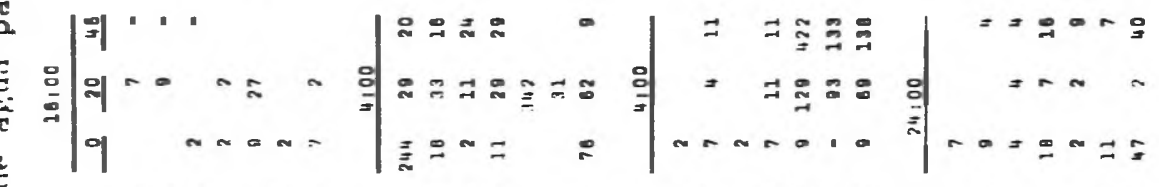

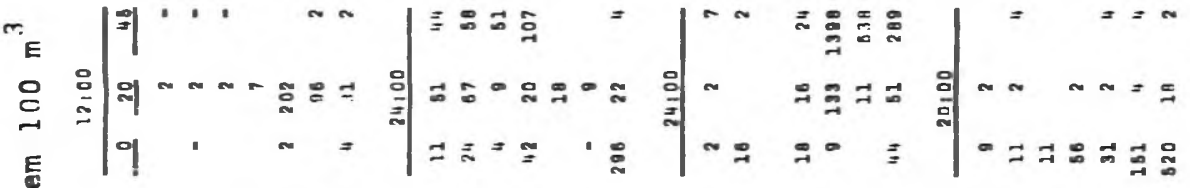

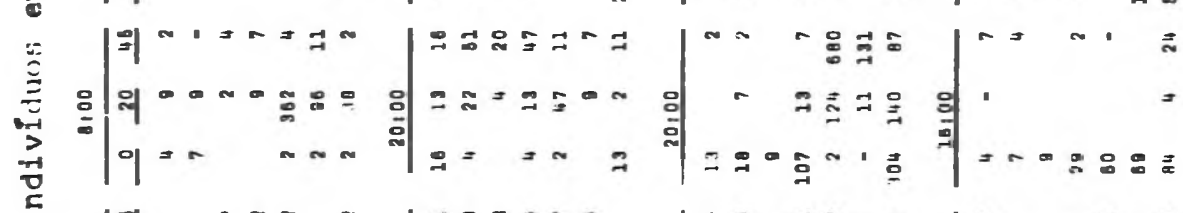

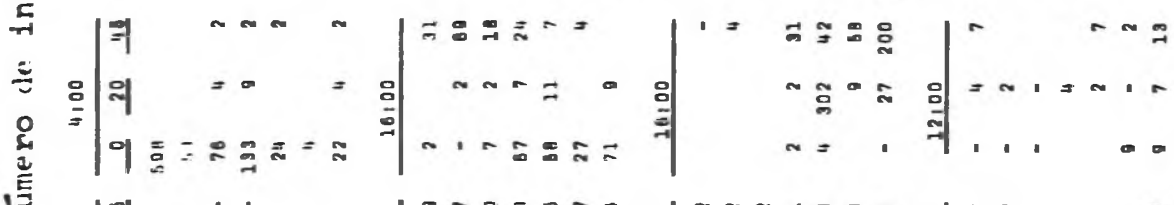

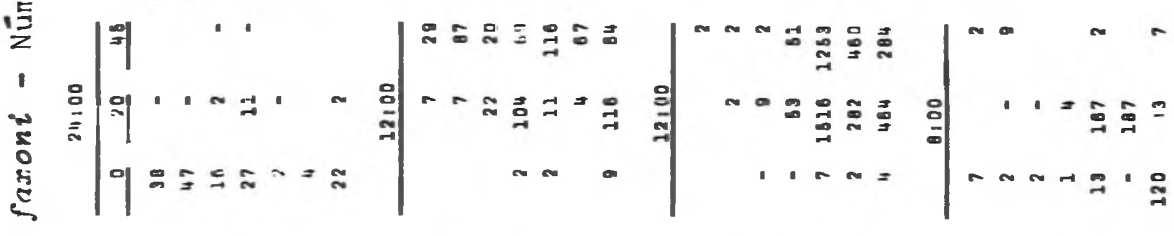

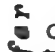

$\lim ^{2} \frac{1}{5}$

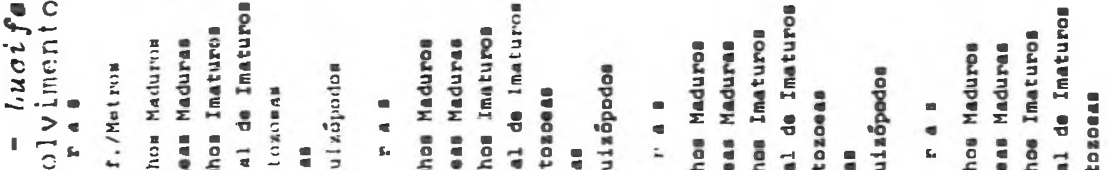

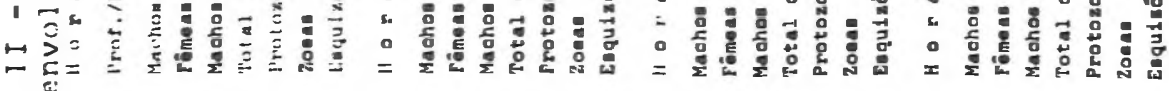

ำ

$09 / 6 / 22$

T9/2/9

T9/TT/L 

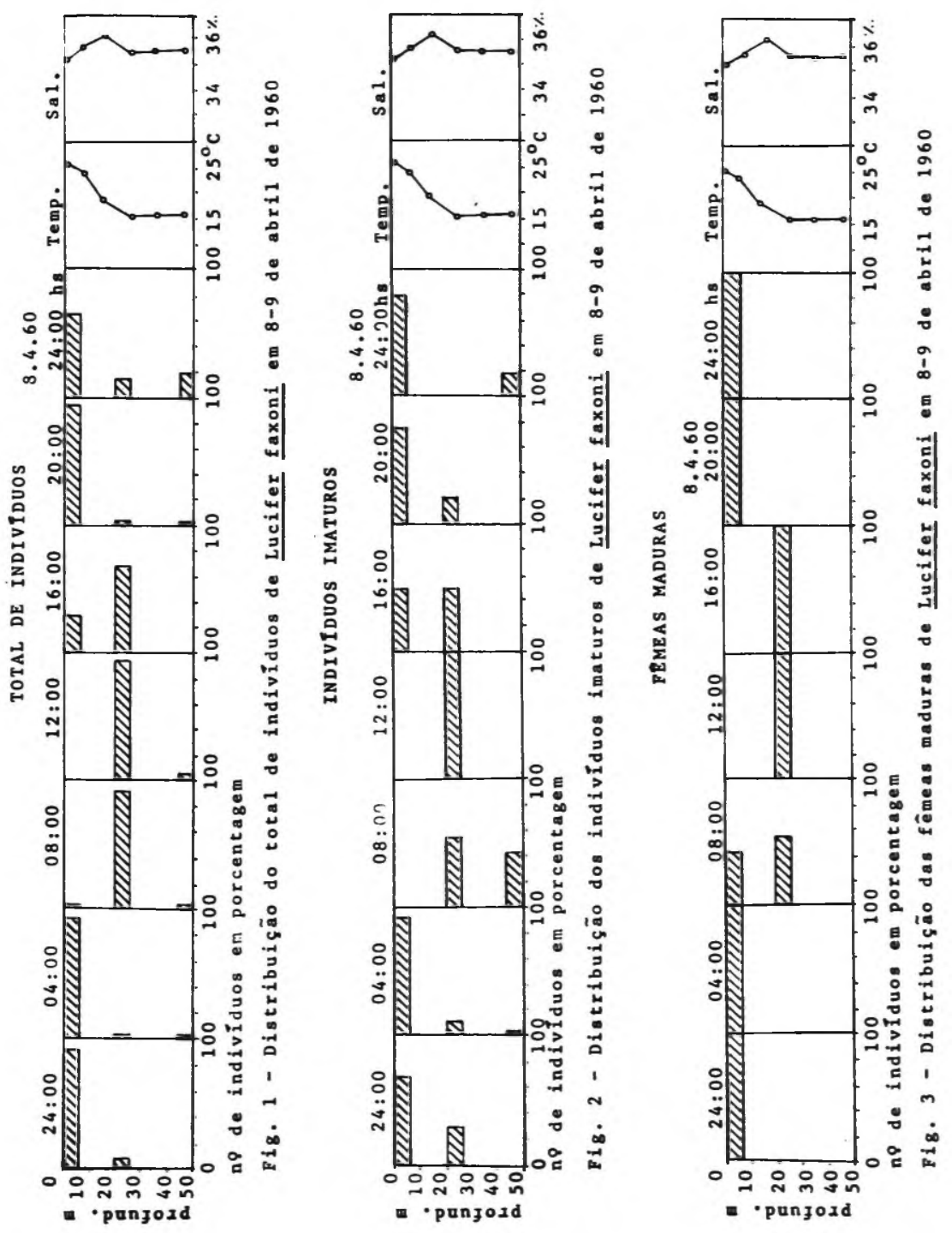

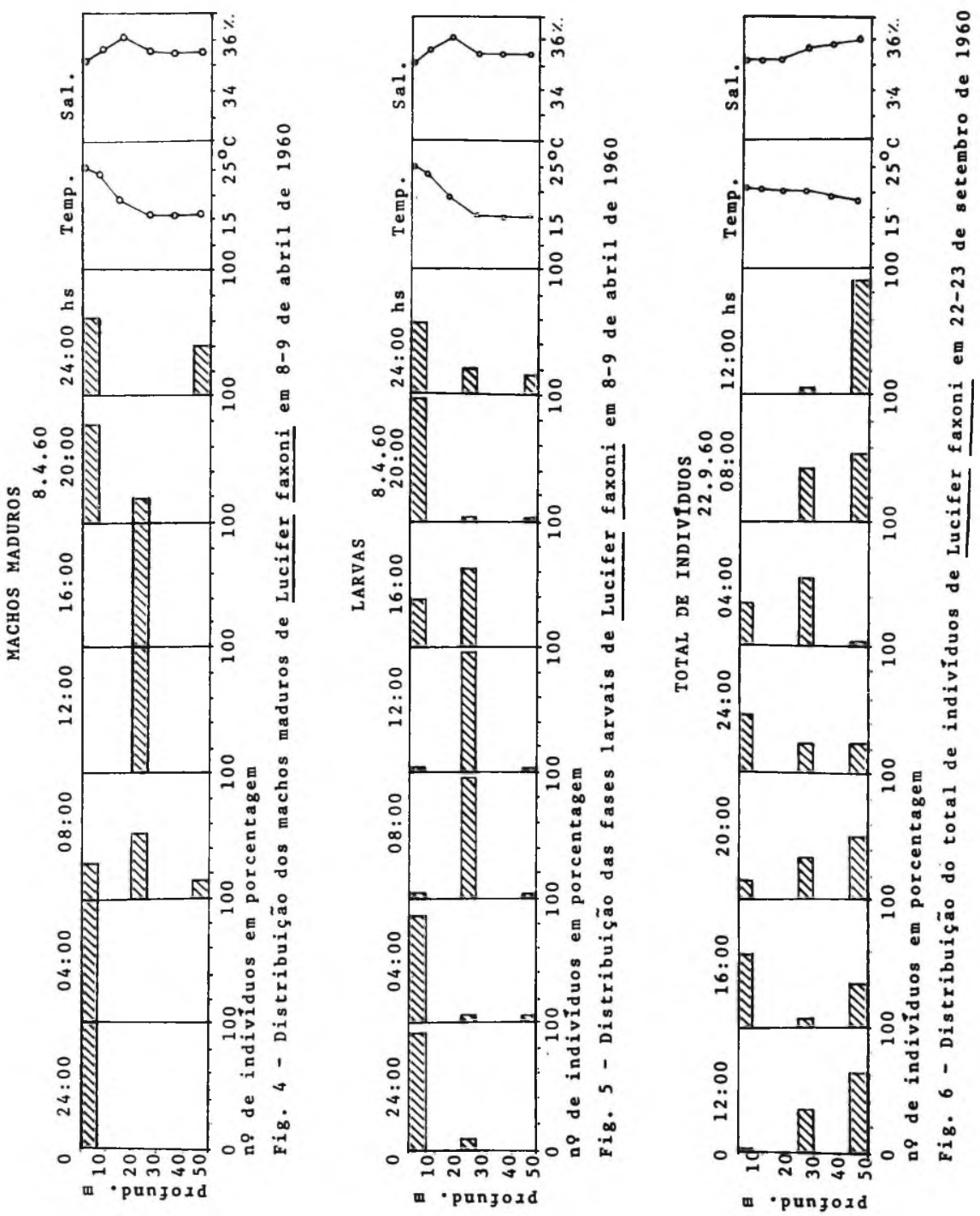

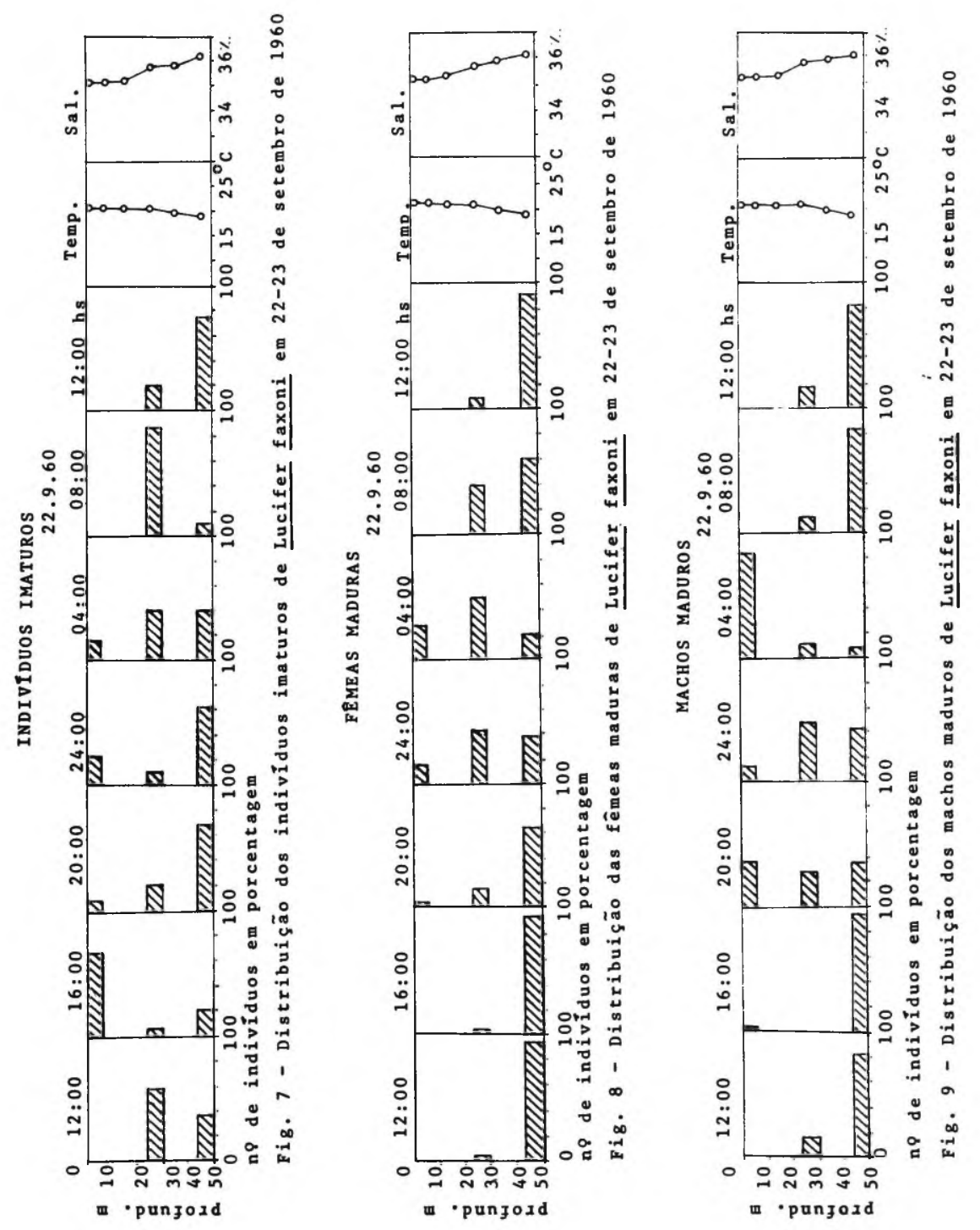

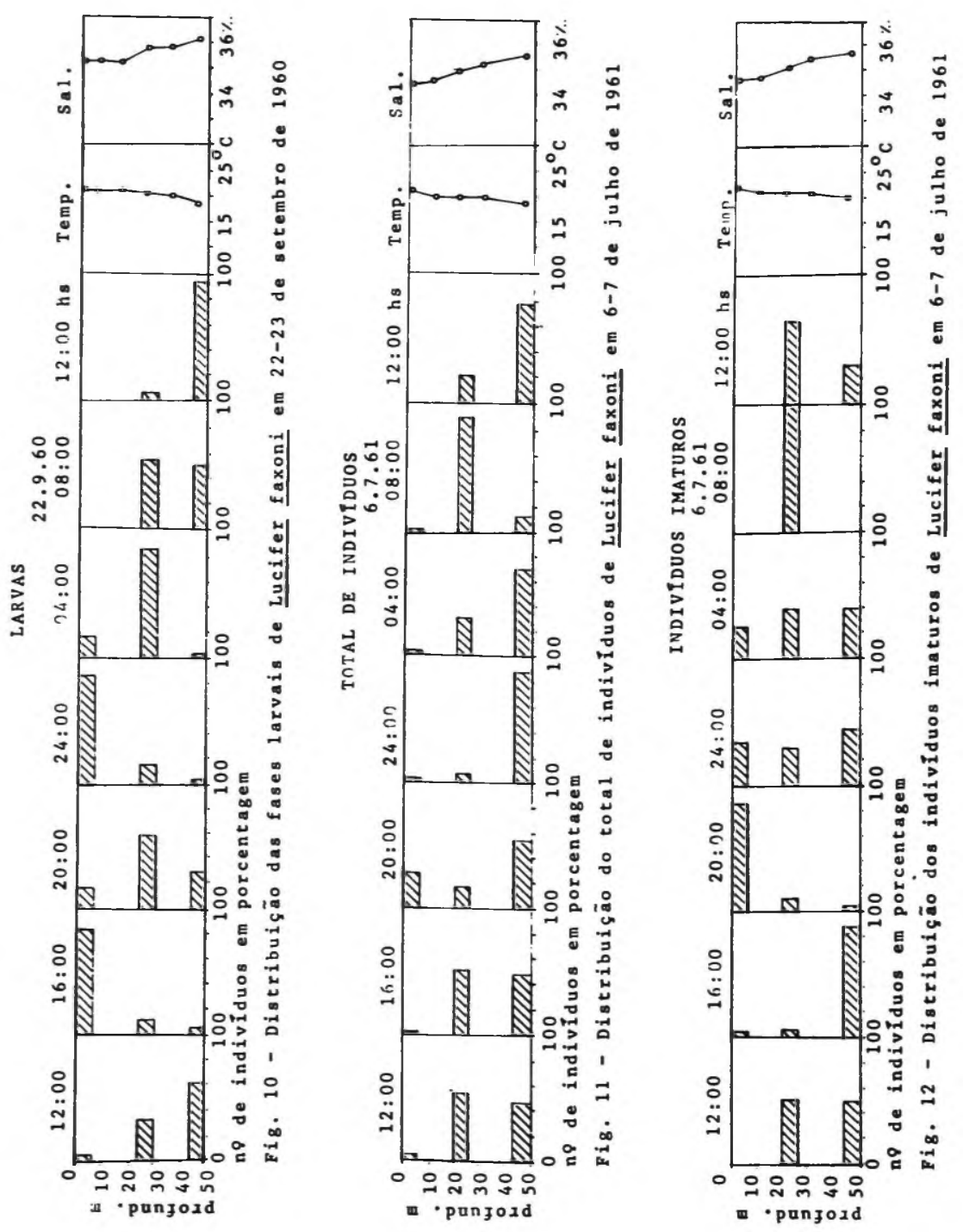

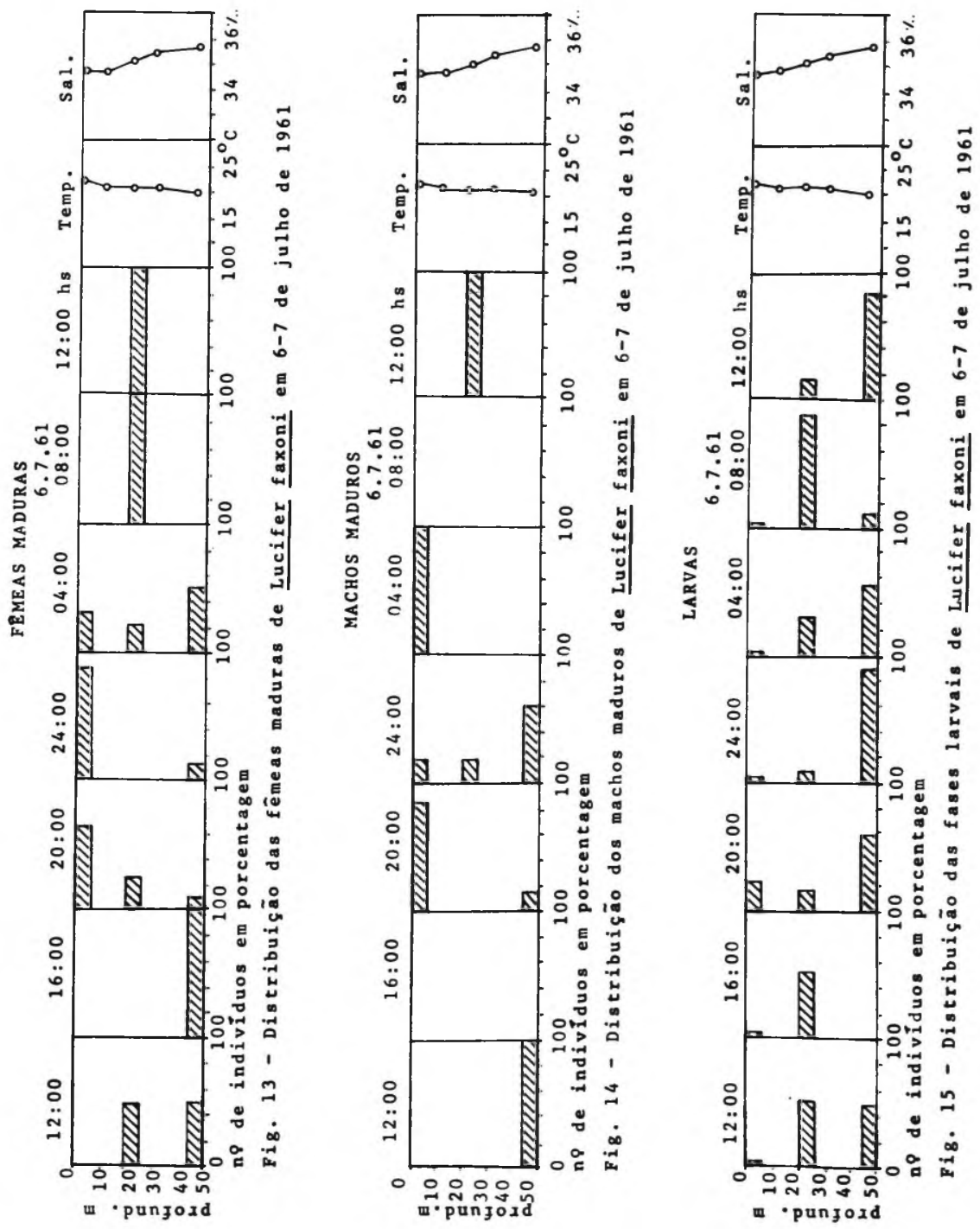

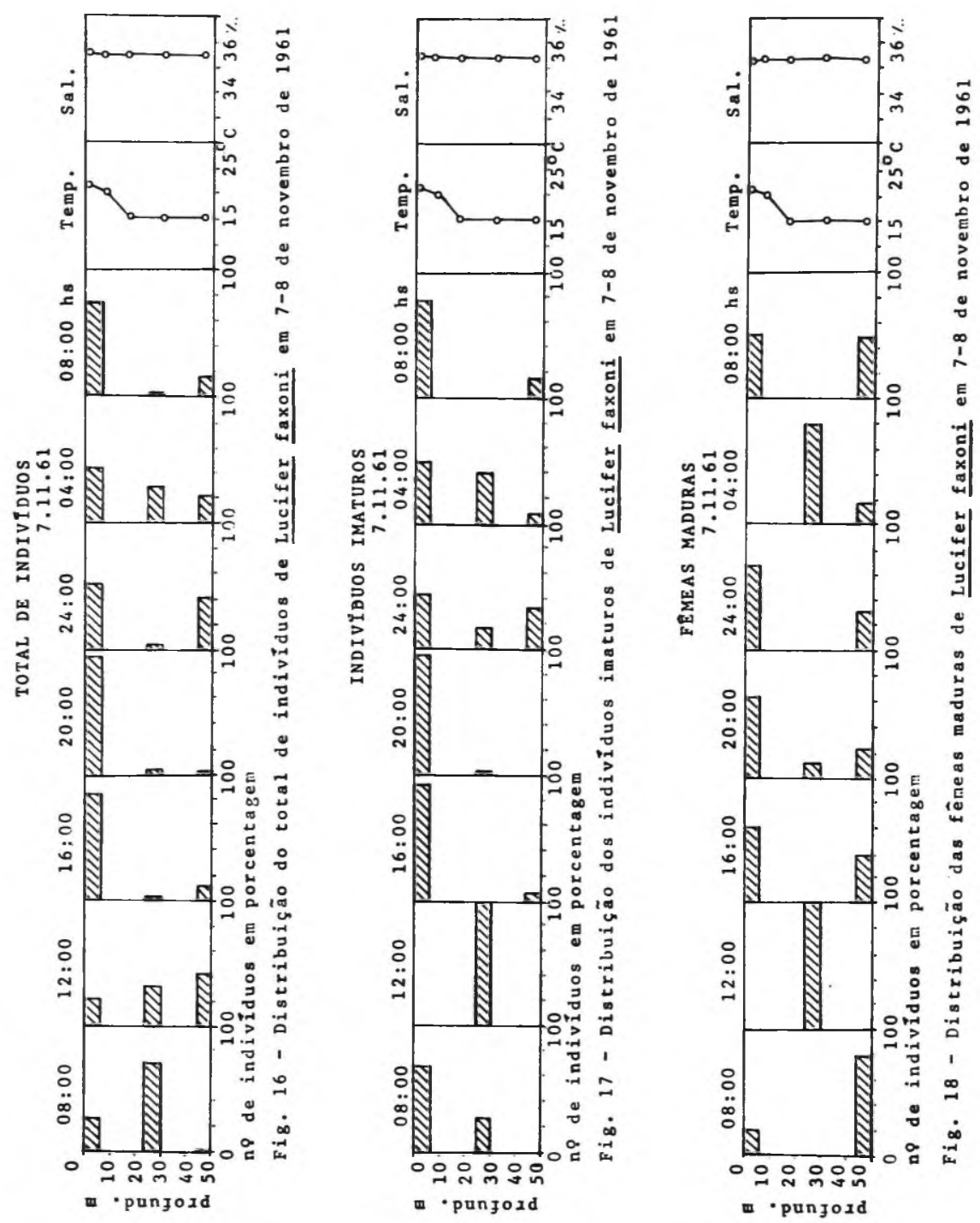

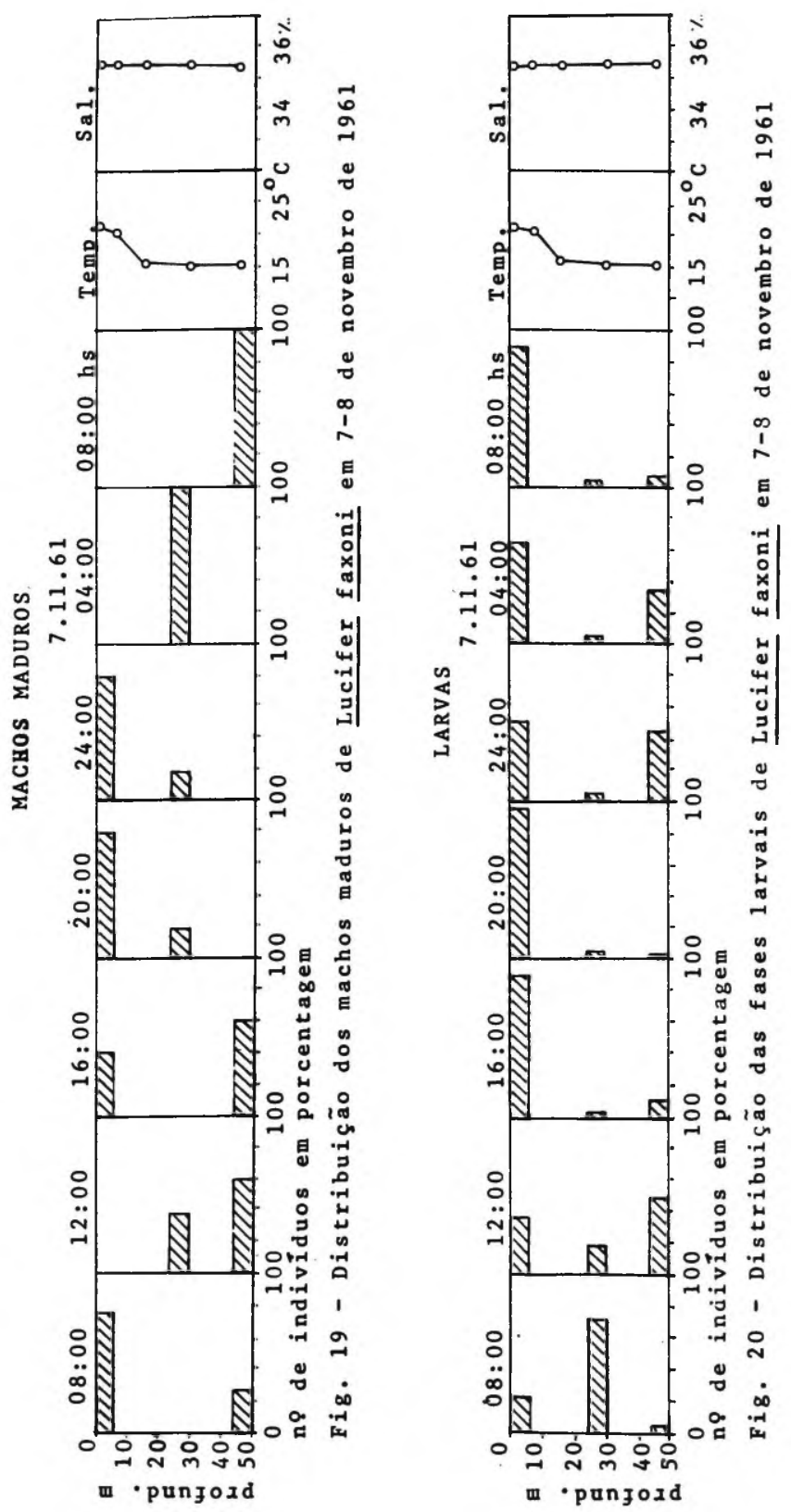
